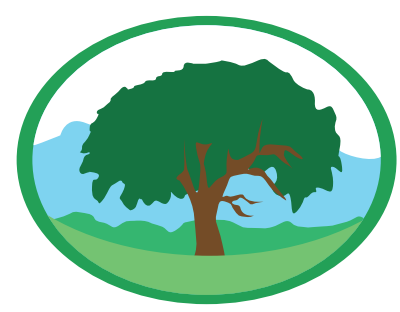

\title{
Automatização do sistema de cálculo de áreas topográficas através do uso combinado de programas de computador
}

\author{
GARRÉ, S. 0. '; WEYMAR, G. J.2; \\ 'Doutorando do Programa de Pós-Graduação em Engenharia Mecânica da Universidade Federal do Rio Grande do Sul \\ ${ }^{2}$ Professor Centro de Engenharias Universidade Federal de Pelotas - CEng/UFPel
}

Palavras-Chave: cálculo de áreas topográficas, interação entre AutoCAD, ArcGIS e Excel, automatização topográfica.

\begin{abstract}
Resumo
0 propósito central deste trabalho foi desenvolver um sistema de interação com o usuário que gerasse a redução do tempo de cálculo de áreas durante o desenvolvimento de mapas topográficos. Para tanto, programou-se uma comunicação entre AutoCAD Map 3D $®$, ArcGIS $®$ e Excel $\circledast$ (versão para estudantes e educadores), a qual possibilitou a troca de informações necessárias para a geração automática da planilha de cálculo. Para avaliar o desempenho da solução apresentada, fez-se um comparativo entre o Método Tradicional e o Método Automatizado. Nestes, para a geração do mapa, o operador separa as áreas (Campo Nativo, Estrada, Área Úmida, Campo Limpo, entre outras) em polígonos individuais que são exportados para o ArcGIS o qual efetua o cálculo da área de cada polígono e informa os valores obtidos. Estes resultados são posteriormente inseridos manualmente (Método Tradicional) ou automaticamente (Método Automatizadol na planilha de cálculos do AutoCAD Map 3D. Nesta análise, feita por meio de cronometragem, concluiu-se que o Método Automatizado reduziu em $67 \%$ o tempo de cálculo. Espera-se um percentual de redução no tempo de cálculo ainda maior, quando o operador trabalhar com mapas topográficos de alta complexidade, uma vez que estes necessitam de maiores detalhamentos e apresentam diversificações de características. Descreve-se todo o procedimento com o intuito de que a solução abordada possa ser utilizada em problemas distintos.
\end{abstract}

\section{Automation of the topographical area calculation system through the combined use of computer programs}

\section{Keywords: calculation of} topographical areas, interaction between AutoCAD, ArcGIS and Excel, topographic automation.

\begin{abstract}
The main purpose of this work was to develop a system of interaction with the user that generated the reduction of the time of calculation of areas during the development of topographic maps. For this purpose, a communication between AutoCAD Map 3D $®$, ArcGIS $®$ and Excel $®$ (version for students and educators) was programmed, which enabled the exchange of information necessary for the automatic generation of the spreadsheet. To evaluate the performance of the presented solution, a comparison was made between the Traditional Method and the Automated Method. In these, for the generation of the map, the operator separates the areas (Native Field, Road, Wet Area, Clean Camp, among others) into individual polygons that are exported to ArcGIS, which calculates the area of each polygon and informs the values obtained. These results are then entered manually (Traditional Method) or automatically (Automated Method) in the AutoCAD Map 3D spreadsheet. In this analysis, made by means of timing, it was concluded that the Automated Method reduced the calculation time by $67 \%$. A reduction percentage is expected in the computation time even greater, when
\end{abstract}


the operator works with topographic maps of high complexity, since these need more detail and presents diversification of characteristics. The whole procedure is described in order that the solution addressed can be used in different problems

\section{INTRODUÇÃO}

A aplicação do processo cartográfico sobre uma coleção de informaçôes, com vistas à obtenção de uma representação gráfica da realidade perceptível, comunicada a partir da associaçáo de símbolos e outros recursos gráficos que caracterizam a linguagem cartográfica é denominada de mapeamento (D’ALGE, 2001; IBGE, 2016).

Carta ou Mapa também é descrito como a representação gráfica sobre uma superfície plana, dos detalhes físicos, naturais e artificiais, de parte ou de toda a superfície terrestre - mediante símbolos ou convençôes e meios de orientação indicados, que permitem a avaliação das distâncias, a orientação das direçóes e a localizaçáo geográfica de pontos, áreas e detalhes -, podendo ser subdividida em folhas, de forma sistemática, obedecido um plano nacional ou internacional. Esta representação em escalas médias e pequenas leva em consideração a curvatura da Terra, dentro da mais rigorosa localização possível relacionada a um sistema de referência de coordenadas. A carta também pode constituir-se numa representaçáo sucinta de detalhes terrestres, destacando, omitindo ou generalizando certos detalhes para satisfazer requisitos específicos. A classe de informaçóes, que uma carta, ou mapa, se propóe a fornecer, éindicada, frequentemente, sob a forma adjetiva, para diferenciaçáo de outros tipos, como, por exemplo, carta aeronáutica, carta náutica, mapa de comunicação, mapa geológico (NBR 13133, 1994; INPE, 2001; VALERIANO, 2016).

Com as novas políticas governamentais do país em relação ao controle e cadastro de áreas, bem como os demais serviços necessários para o perfeito mapeamento de regióes específicas, surgiram diversas demandas no ramo de topografia. Esse detalhamento permite, por exemplo, um maior controle sobre áreas degradadas, cultivadas, construídas, entre outras. Além de também poder possibilitar uma maior facilidade na hora da escolha de áreas para alocar obras e/ou outros tipos de atividades(MOURA, 2003; FILHO et al,
2006; MDA, 2016).

Geralmente a caracterizaçáo de uma determinada área é feita pelo auxílio de mapas. Esses mapas são gerados após o levantamento a campo e evidenciam os componentes presentes na área total de uma propriedade. Neles são descritos os tipos de vegetação, tipo de relevo, Áreas de Preservação Permanente (APP), corpos hídricos, áreas cultivadas, áreas com construçōes, entre outras características (BUENO, 2009).

Além do corpo técnico, que faz o levantamento a campo, a geração de mapas também necessita de operadores que, entre outras coisas, dominem software CAD (Computer Aided Design).

Tendo em vista que a confecçáo de um mapa topográfico demanda horas de dedicaçáo, o intuito central desse trabalho é reduzir o tempo gasto com o projeto a partir do momento que os dados chegam no escritório e são repassados aos projetistas.

Para isso desenvolve-se uma interação entre três tipos de software (AutoCAD Map 3D, ArcGIS e Excel) a qual permite a redução do tempo de cálculo gerando a reduçáo de custos e otimiza os ganhos pelo maior número de mapas confeccionados num mesmo intervalo de tempo.

\section{MATERIAL E MÉTODOS}

\section{Local de desenvolvimento dos estudos}

Este trabalho foi desenvolvido em uma empresa situada na cidade de Pelotas, Rio Grande do Sul, Brasil a qual tem suas atividades voltadas para o georeferenciamento de imóveis, cartografia, topografia, geodesia, geoprocessamento e fotogrametria. Atualmente seus funcionários estáo divididos em áreas como processamento de dados, gerenciamento, confecção de mapas e serviço de coleta de dados, ou seja, serviço de campo. Com a soma das diferentes áreas de atuaçáo promove o Georeferenciamento de pequenas e grandes áreas para várias finalidades, entre elas destacam-se: Certificação e atualização do Certificado de Cadastro de Imóvel Rural (CCIR) junto ao Instituto Nacional de Colonização e Reforma Agrária (INCRA), parcelamento do solo, transferência de titularidade, retificação, inventário, averbaçáo de 
reserva legal, meio ambiente dentre outros (INCRA, 2016).

A seguir, no subitem 2.2, apresenta-se a metodologia seguida pela empresa para geração de mapas.

A solução proposta nesse artigo foi executada em ambiente externo à empresa a qual não possuía computador disponível para essa finalidade pela alta demanda de serviços e, portanto, todos os tipos de software citados foram utilizados em sua versão para estudantes e educadores.

\section{Metodologia de trabalho anterior a automatização}

Primeiramente faz-se o levantamento a campo. Os dados obtidos são transferidos para o computador e começam a ser tratados. Com esses dados registrados em software CAD (AutoCAD Map 3D ${ }^{\circledast}$ ) é então projetado um mapa(planta base)que possibilita o início da planta topográfica detalhada. A planta base é dotada apenas do perímetro, lindeiros e do número e nome dos marcos adotados a campo (Figura 1).

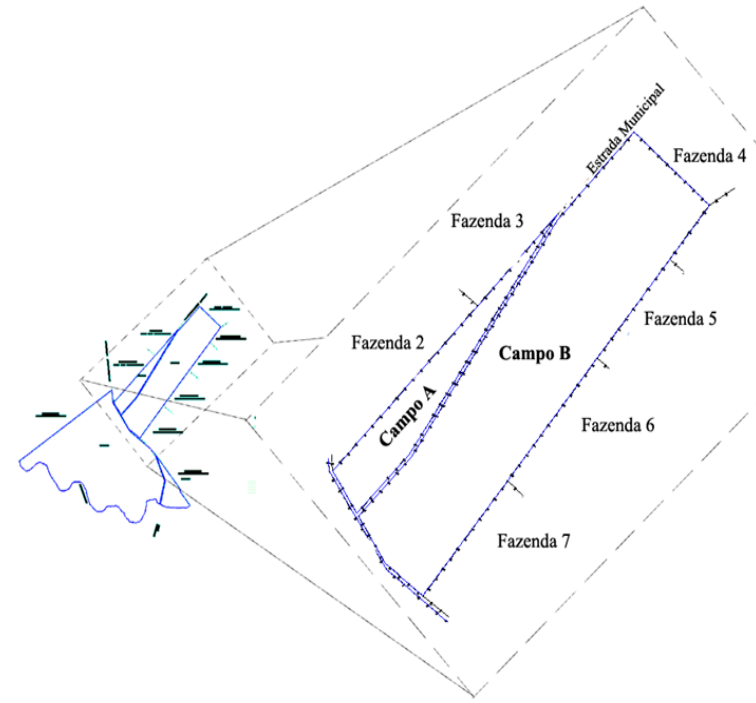

Figura 1. Detalhes da planta base

Após essa etapa concluída, a planta detalhada começa a ser processada. Esse detalhamento consiste na inserção de imagens (coletadas do Google Earth ${ }^{\circledR}$ ou adquiridas através de fotografias aéreas) na área de trabalho do AutoCAD.

Uma vez ajustadas, ou seja, georeferenciadas, iniciase o mapa de detalhamento da propriedade (Figura 2) aliando-se as informaçóes coletadas a campo com a interpretação das imagens.
Enfim divide-se a área total em campos (A,B,C,D,..) e fazo detalhamento descrevendo todas as características da propriedade. Feito o detalhamento, os polígonos gerados por cada área são exportados para o ArcGIS. Para tanto, no AutoCAD, deve-se selecionar um único layer e desativar-se os demais (Figura 3).

Repete-se os passos para todos os polígonos de cada campo salvando-os em pastas individuais, conforme exposto na Figura 4A, Figura 4B eFigura 4C, com a finalidade de facilitar a ligação entre arquivos e extensóes.

O AutoCAD, no processo de exportação, salva os arquivos em três extensões diferentes, Arquivo $D B F$, AutoCAD Shape Source e AutoCAD Compiled Shape. Estas extensões, são as responsáveis pela comunicação entre AutoCAD, ArcGIS e Excel. E através de sua manipulação é possível automatizar o processo de cálculo das áreas (abordado no subitem 2.3).

O ArcGIS se encarrega de efetuar os cálculos de cada polígonoautomaticamente após a exportação. Os valores informados por esse software, são digitados na tabela do AutoCAD (Tabela Geral), a qual é desenhada conforme exposto na Figura 5 e tem seus valores de resultados totais e percentuais calculados por auxílio de calculadora, o que pode aumentar a chance de erro e, em muito, o tempo de cálculo.

O ArcGIS calcula a área dos polígonos gerados no AutoCAD, os quais necessariamente precisam ter sido separados em layers com nomes diferentes. Por exemplo, mata nativa (layer - mata nativa), Açude (layer açude), entre outros.

Para dar início ao processo de cálculo abre-se o ArcGIS, clica-se em inserir um novo polígono (botão Add Data em destaque na Figura 6A) e na aba que se apresenta (Figura 6B) escolhe-se o polígono (gerado previamente no AutoCAD) que terá sua área calculada.

Depois de inserido o novo polígono insere-se um novo campo (Add Field) seguindo o exposto na Figura 7A e $7 \mathrm{~B}$.

Após nomear-se a coluna deve-se determinar quantas casas decimais se deseja após a vírgula através da opção Type (neste caso opta-se por Double (até quatro casas decimais após a vírgula)) (Figura 8A).

Em seguida, opta-separa efetuar o cálculo dos 


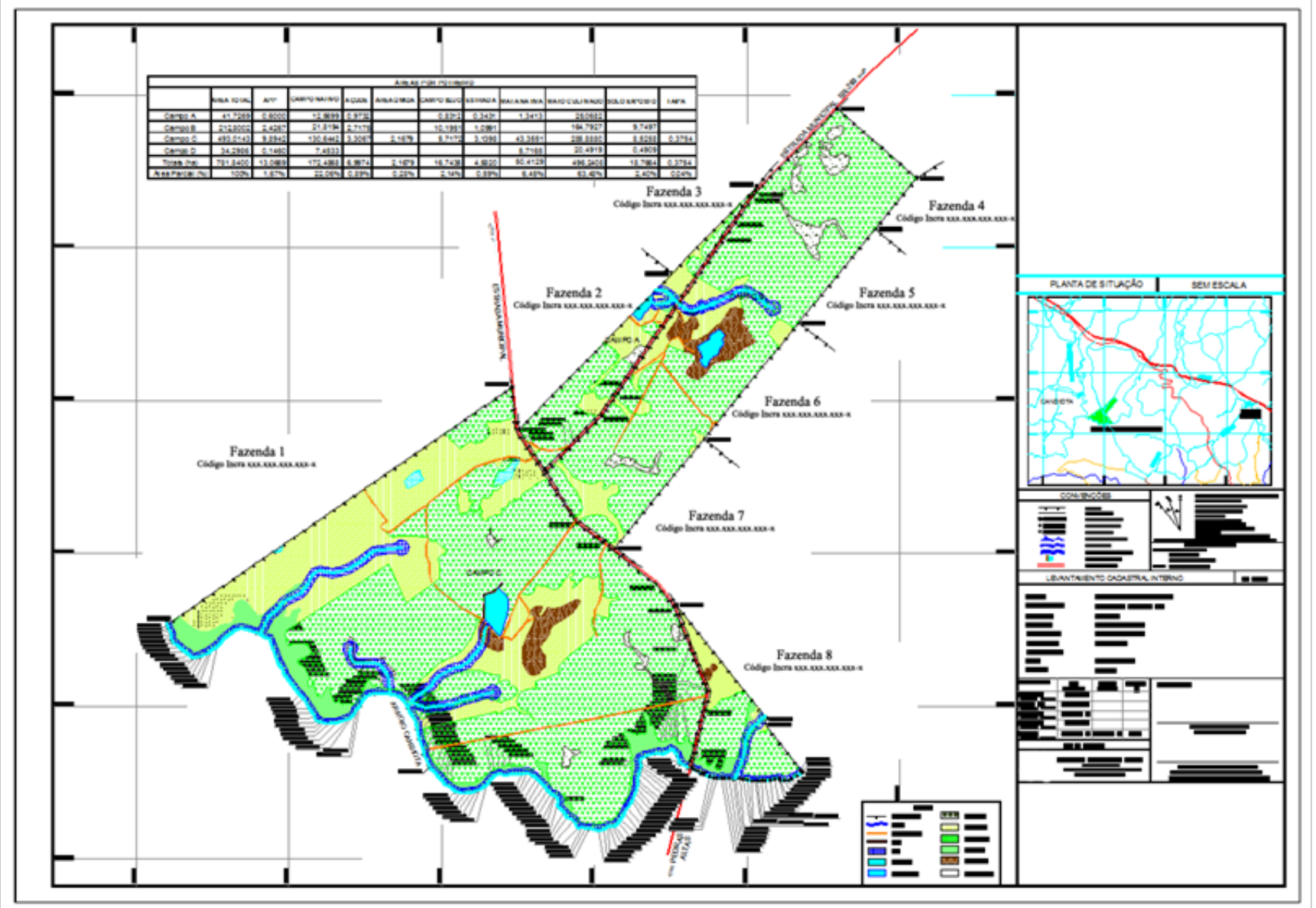

Figura 2. Layout do mapa tradicional finalizado

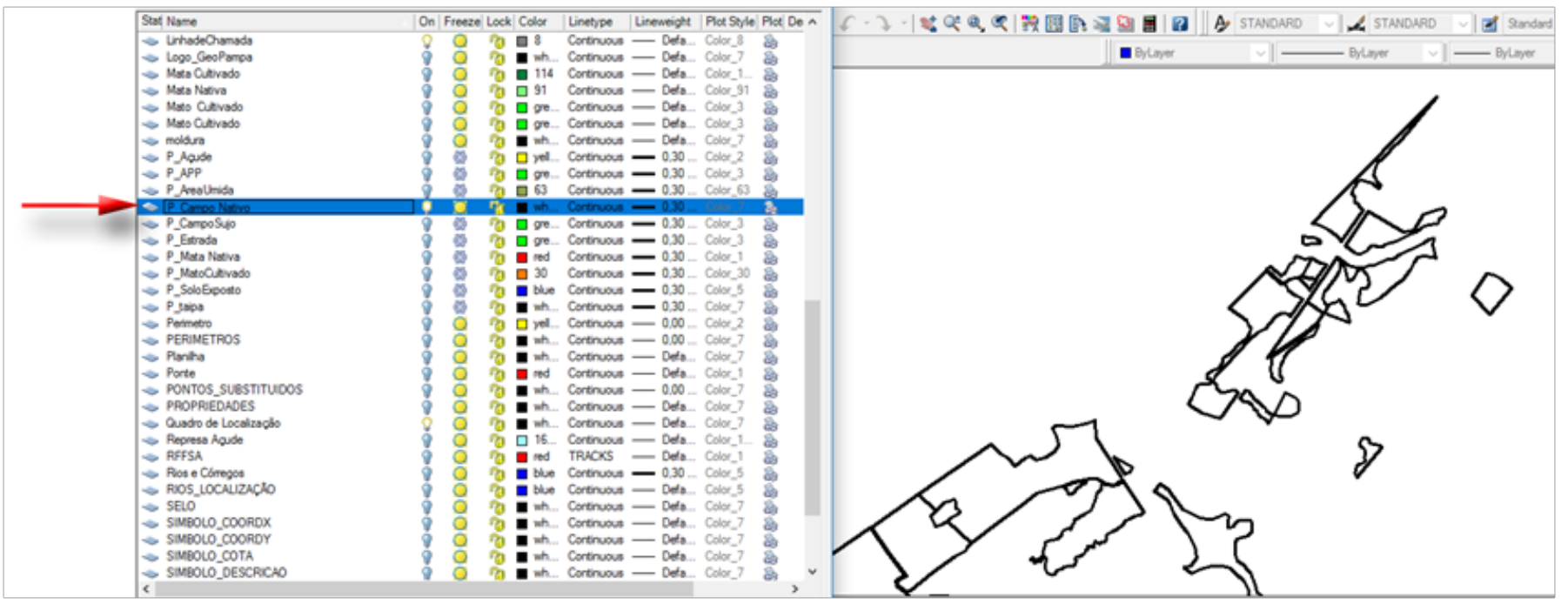

Figura 3. Um único layer selecionado

polígonos opta-se por Field Calculator (Figura 8B). Vale ressaltar que para esse caso a opção escolhida foi a Field Calculator, no entanto em alguns casos pode ser mais prático optar-se pela opção Calculate Geometry, lembrando que para cálculos avançados a Field Calculator é a opção mais indicada (ArcGIS/ArcMap,
2016).

Depois de escolhida a opção que efetuará o cálculo da área uma nova caixa de diálogo se abrirá, nela escolhe-se o nome da coluna anteriormente criada (área1). Aplica-se um duplo clique sobre o nome área 1 e após um clique simples no botão Load (Figura 8C). 

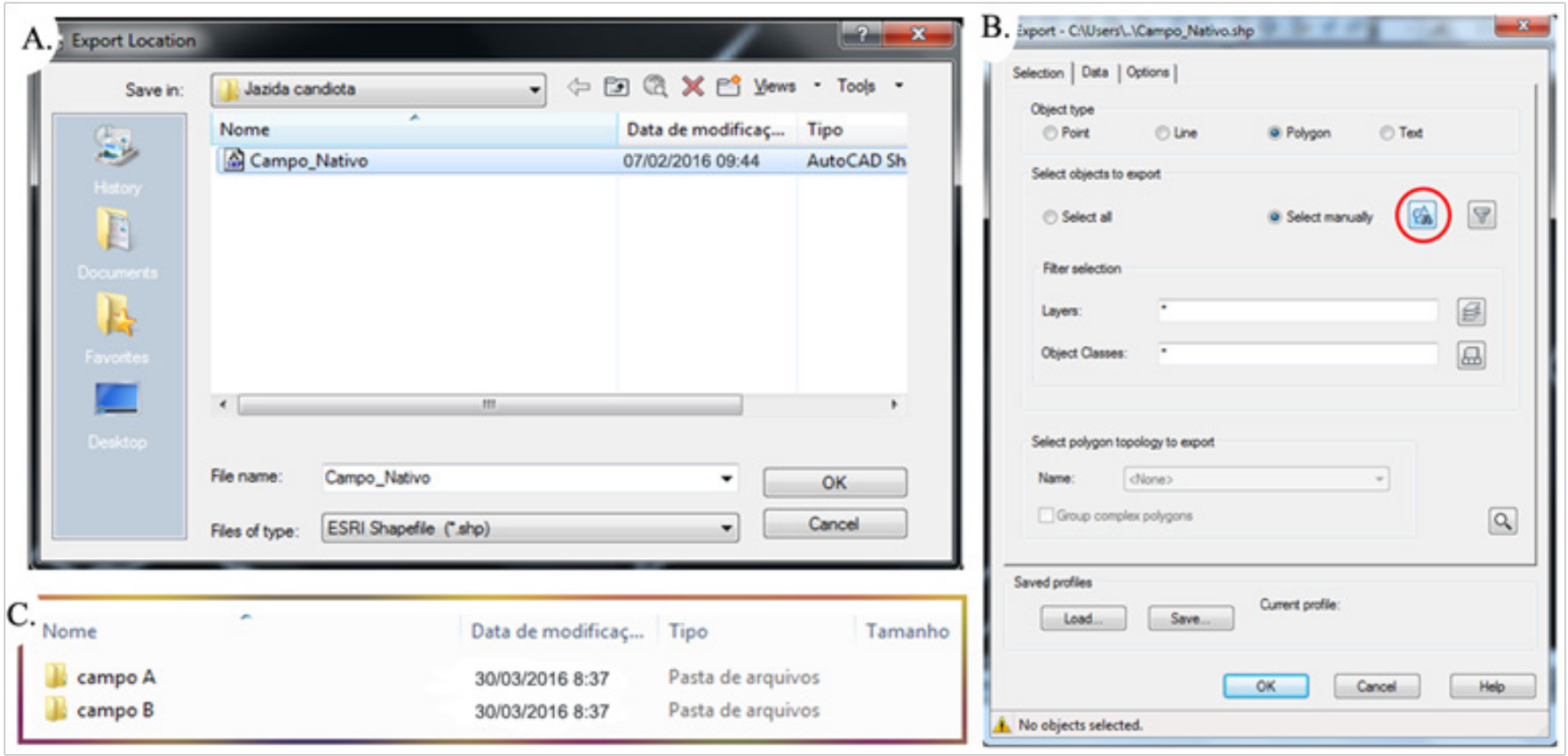

Figura 4. Procedimento para exportação dos polígonos no AutoCAD: A. Caixa de opções de salvamento; B. Botão para acionar a função selecionar; e C. Modelo de pastas individuais

\begin{tabular}{|c|c|c|c|c|c|c|c|c|c|c|c|}
\hline \multicolumn{12}{|c|}{ ÁREAS POR POTREIRO } \\
\hline & AREA TOTAL & APP & CAMPO NATIVO & AÇUDE & ÁREA ÚMIDA & CAMPO SUJO & ESTRADA & MATA NATIVA & MATO CULTIVADO & SOLO EXPOSTO & TAIPA \\
\hline Campo A & 41,7269 & 0,6000 & 12,5699 & 0,9732 & & 0,8312 & 0,3431 & 1,3413 & 25,0682 & & \\
\hline Campo B & 212,8002 & 2,4267 & 21,8194 & 2,7175 & & 10,1951 & 1,0991 & & 164,7927 & 9,7497 & \\
\hline Campo C & 493,0143 & 9,8942 & 130,6442 & 3,3067 & 2,1679 & 5,7172 & 3,1398 & 43,3551 & 285,8880 & 8,5258 & 0,3754 \\
\hline Campo D & 34,2986 & 0,1460 & 7,4533 & & & & & 5,7165 & 20,4919 & 0,4909 & \\
\hline Totais (ha) & 781,8400 & 13,0669 & 172,4868 & 6,9974 & 2,1679 & 16,7435 & 4,5820 & 50,4129 & 496,2408 & 18,7664 & 0,3754 \\
\hline Área Parcial (\%) & $100 \%$ & $1,67 \%$ & $22,06 \%$ & $0,89 \%$ & $0,28 \%$ & $2,14 \%$ & $0,59 \%$ & $6,45 \%$ & $63,48 \%$ & $2,40 \%$ & $0,04 \%$ \\
\hline
\end{tabular}

Figura 5. Layout da tabela gerada manualmente no AutoCAD

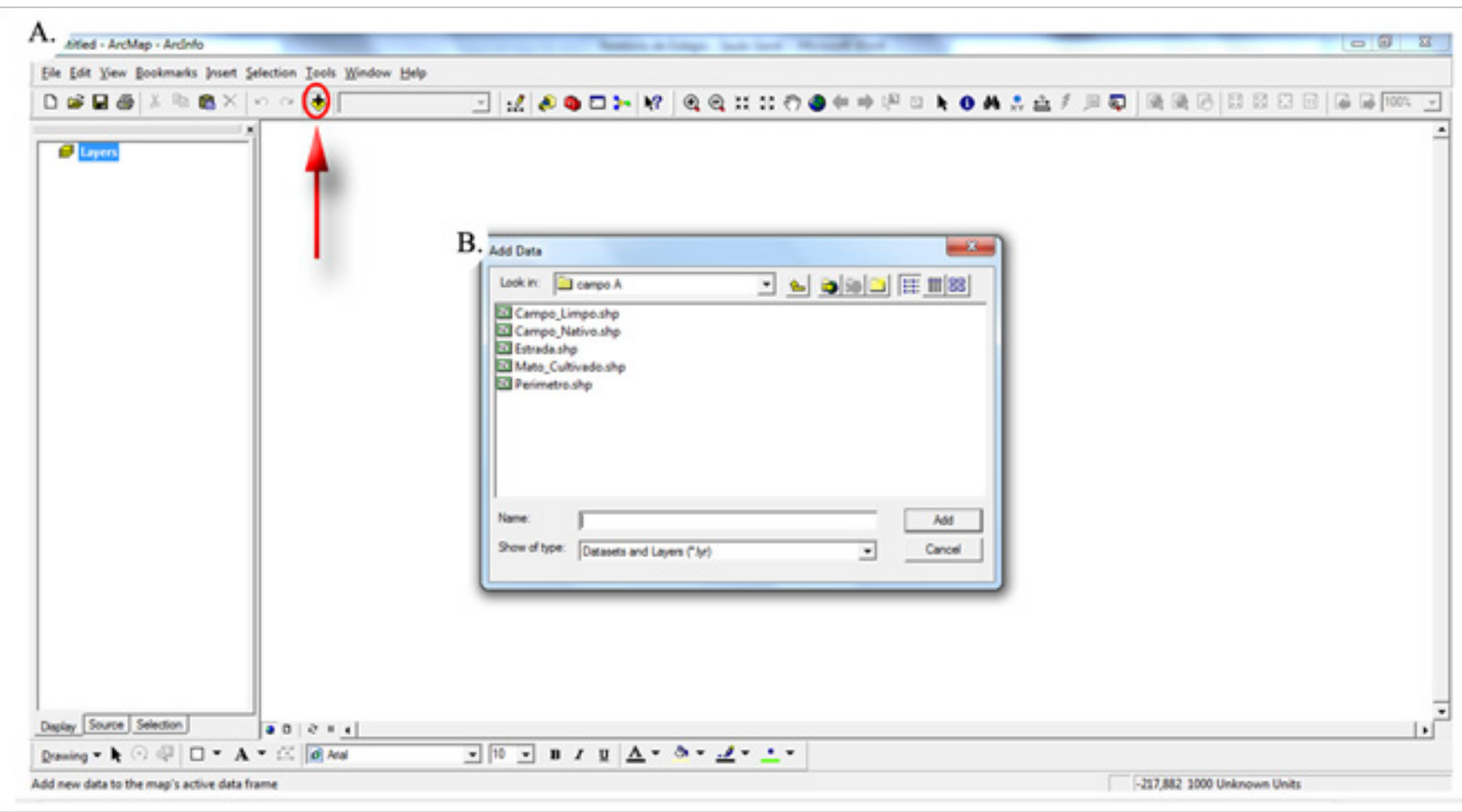

Figura 6. Início do processo de cálculo no ArcGIS: A. Botão para inserir novo polígono; e B. Caixa de diálogo para selecionar e adicionar novo polígono 
A. $\overline{\text { lagers }}$

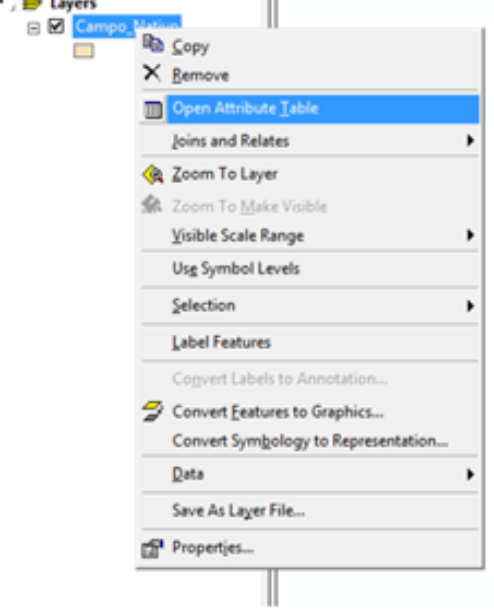

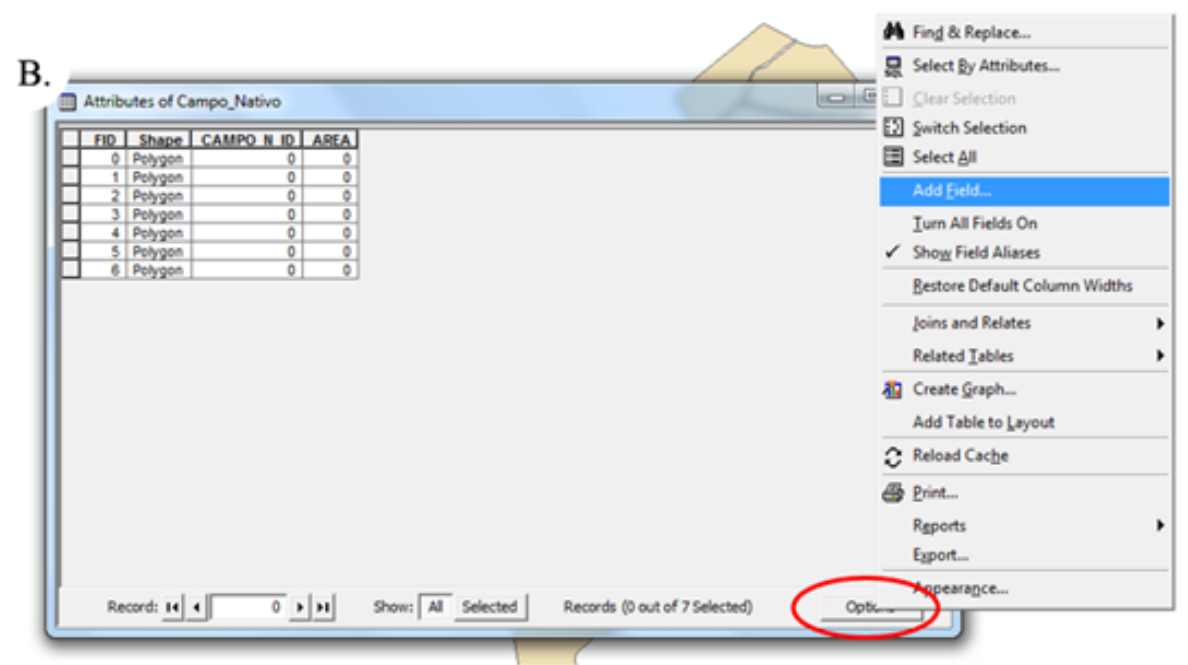

Figura 7. Procedimento de cálculo das áreas de polígonos através do ArcGIS (Parte I): A.Open Attribute Table; eB.Options

A.

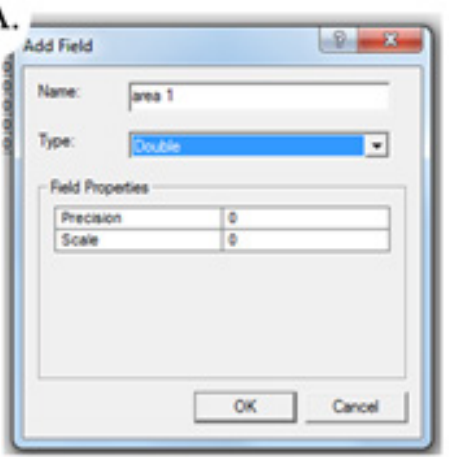

B.

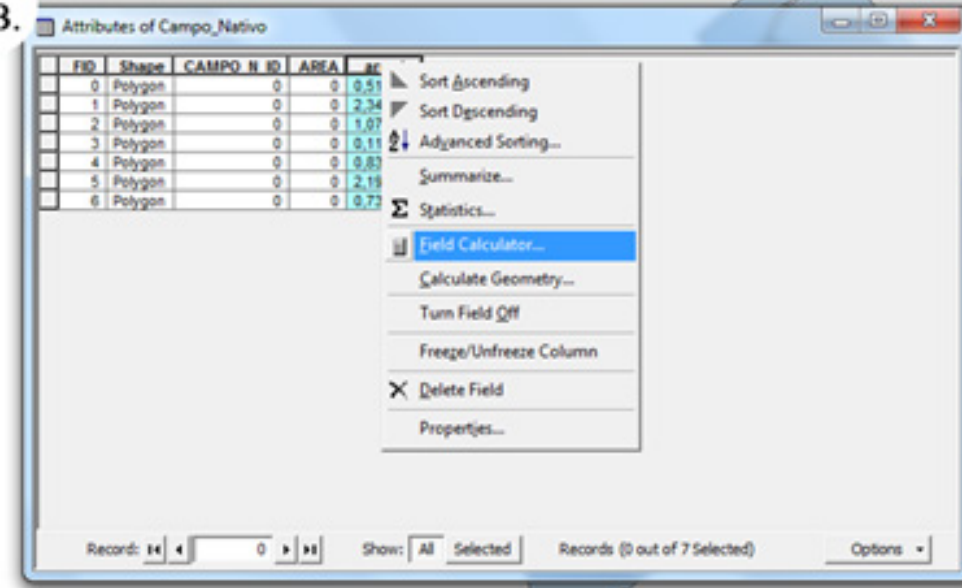

C.

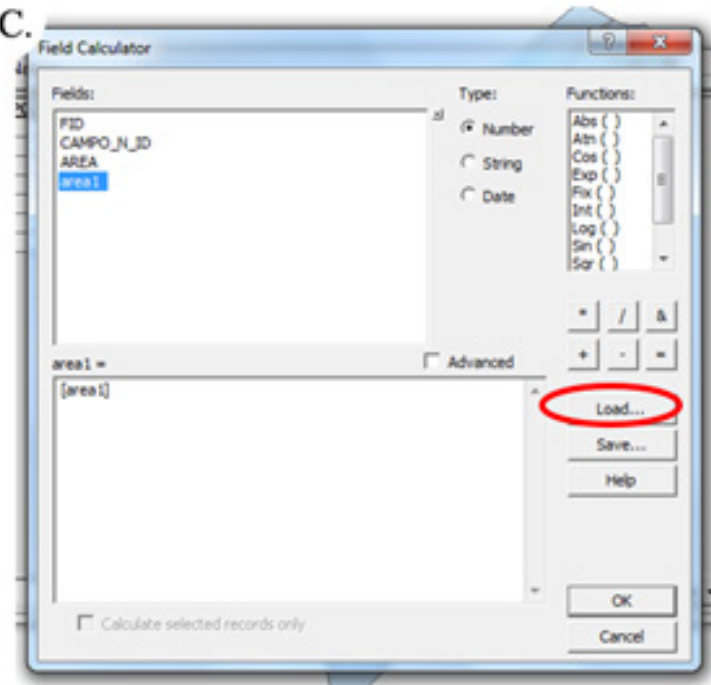

D.

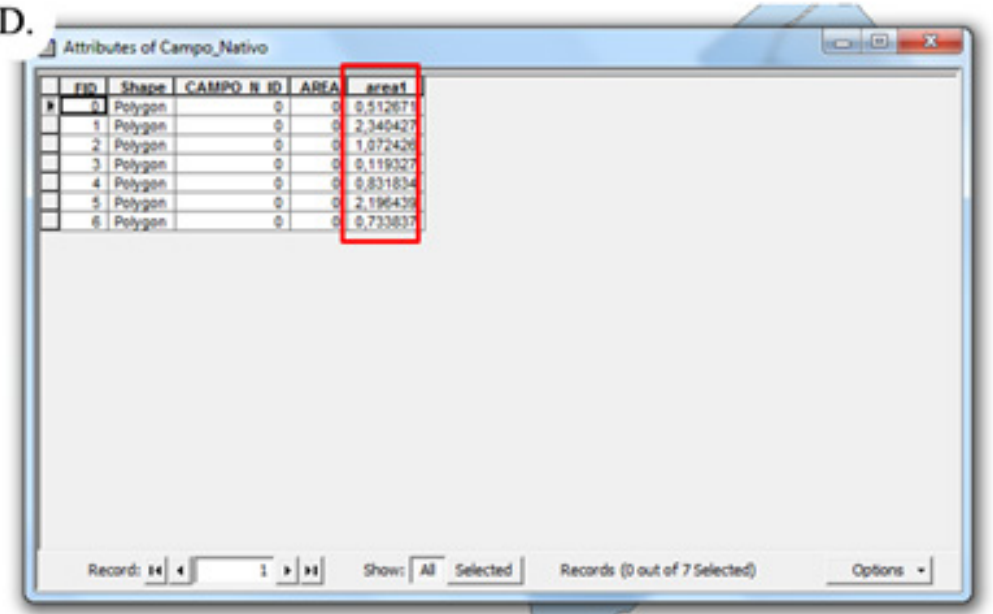

Figura 8. Procedimento de cálculo das áreas de polígonos através do ArcGIS (Parte II): A.Type: Double; B.Field Calculator; C.Load, e D.area1 (áreas calculadas) 

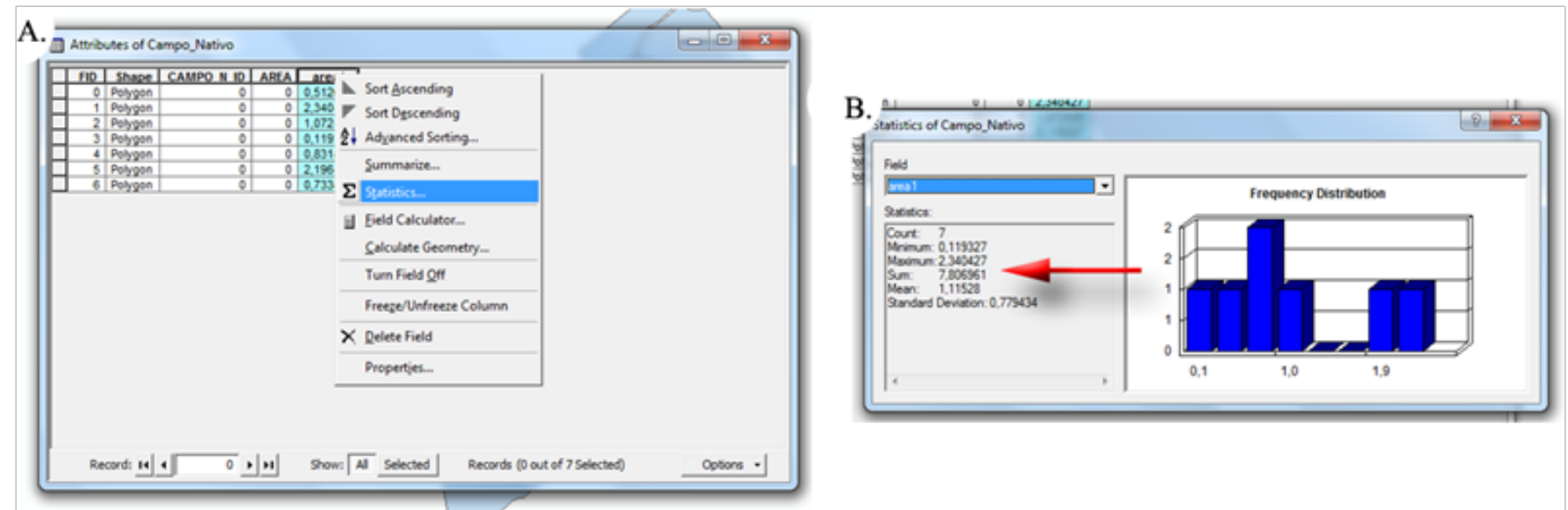

Figura 9. Procedimento para se obter a área total dos polígonos: A.Statistics; e B. Área final resultante do somatório de todos os polígonos e estatísticas

Feito isso, o ArcGISse comunica com suas expressóes. Nas expressões existem diversas formas de cálculo (área em hectares, em metros quadrados, cálculo do perímetro, entre outras).

Para o caso desse estudo escolhe-se a opção Área ha, pois assim o resultado obtido na coluna (área1) estará em hectare. Após isso clica-se em abrir e observa-se o resultado na coluna criada (Figura $8 \mathrm{D}$ ).

Após o programa calcular a medida da área individual de cada polígono, ele ainda poderá fornecer a soma geral deles. Para tanto, clica-se novamente com o botão direito sobre a coluna área1. $\mathrm{Na}$ caixa que se abre clica-se em Statistics(Figura 9A) e tem-se a área final resultante do somatório de todos os polígonos (Figura 9B).

Para finalizar o procedimento de cálculo, os dados gerados pelo ArcGIS são inseridos manualmente na tabela do AutoCADa qual descreve as áreas características de cada propriedade conforme explicitado, anteriormente, na Figura 5. E finalmente são calculados, com auxílio de calculadora, os seguintes termos: Totais em ha (calculado pelo somatório de cada Campo) e Área Parcial (calculado pela razão entre o total individual de cada área e o total encontrado na coluna Área total).

No decorrer de dois meses de análise da metodologia de trabalho da empresa nota-se que o processo de cálculos apresenta um padrão, com uma alteração pequena no número de variáveis que caracterizam as áreas de uma propriedade, como por exemplo, Campo Nativo, Mata Nativa, Área de Preservação Permanente, entre outras. Assim surge a possibilidade de automatizar o processo de cálculo. A solução mais prática encontrada, é gerar uma interação entre o AutoCAD, ArcGIS e Excel. O modo como é desenvolvido esse sistema de integração é apresentado no subitem 2.3.

\section{Processo de interação - Automatização do procedimento}

Como já foi salientado no subitem 2.2, todo o trabalho começa no AutoCAD. Lá exportam-se os polígonos gerados e nesse processo o software transforma os polígonos em extensóes reconhecidas pelo ArcGIS. No ArcGIS importam-se os polígonos e calculam-se suas respectivas áreas.

Até esse ponto a metodologia continua a mesma desenvolvida pela empresa. No entanto o diferencial para o processo automatizado é que os valores de área de cada polígono obtidos no ArcGIS não são mais inseridos manualmente na planilha do AutoCAD. Agora vincularam-se os resultados de cálculo do ArcGIS ao Excel que por sua vez foi vinculado ao AutoCAD. Sendo assim, programou-se o Excel para que recebesse os dados do ArcGIS e executasse os cálculos necessários. Em seguida programou-se a ligação do AutoCAD com o Excel para que os valores expressos na Tabela Geral (inserida no AutoCAD) se modificassem automaticamente (GEOBRAINSTORMS, 2016; CADBLOG, 2016).

Para viabilizar esse tipo de processo, faz-se necessário saber que ao exportarem-se os polígonos do AutoCAD, são geradas três extensões diferentes (já mencionadas anteriormente). Uma dessas extensóes é a .dbf, a qual é 
reconhecida pelo Excel. Após exportado do AutoCAD esse tipo de arquivo, se aberto,apresenta uma planilha sem valores (em branco). Isso ocorre, porque antes de abri-lo é necessário que se calcule as áreas no ArcGIS. Após um certo polígono, exportado do AutoCAD, passar pelo processo de cálculo de área do ArcGIS ele altera automaticamente os dados do arquivo.dbfe informa os valores de cada parcela (poligonal) que em seguida serão somadas para gerar a área total de um conjunto de polígonos (de Campo_Nativo, por exemplo).

Note-se aqui que essa planilha corresponde a apenas uma coluna (Campo_Nativo) da Tabela Geral que será inserida no AutoCAD. Para o preenchimento das demais colunas, que apresentam as características de uma dada propriedade, e construção da Tabela Geral deve-se proceder exportando-se do AutoCAD todos os polígonos referentes a cada área (Campo Nativo, APP, Campo Sujo, assim por diante), calculando-se suas respectivas áreas no ArcGIS que, por conseguinte,substitui todas as tabelas individuais (arquivos.$d b f$ com os valores das áreas calculadas).

Em seguida a isso faz-se necessário ir na pasta de salvamento dos arquivos . $d b f$ (onde deverão estar salvos os demais arquivos conforme visto anteriormente $\mathrm{e}$ ilustrado na Figura 4) e abrir todas as tabelas geradas após o cálculo das áreas.

Após abrir-se as tabelas geradas pelo ArcGIS, devese criar um novo documento no Excel e começar a ligar cada célula desse documento (Tabela Geral) com o respectivo resultado obtido nas tabelas calculadas pelo ArcGIS. Para se fazer essa ligação insere-se, no local onde o resultado será apresentado (célula da Tabela Geral), o comando:=Campo_Nativo.dbf $\$ C \$ 2$, por exemplo.

Aqui é válido ressaltar que na célula do novo documento, o qual se tornará a Tabela Geral, se coloca apenas o sinal de igual e na tabela gerada pelo ArcGIS seleciona-se o valor desejado. Para as próximas colunas fazem-se os mesmos procedimentos até que os dados das planilhas individuais estejam na sua totalidade inseridos na Tabela Geral. Concluído isso,programase a Tabela Geral, para que comece a calcular os resultados totais e percentuais.

Para facilitar a compreensão será utilizado um mapa com dois campos - Campo A e Campo B - com seis colunas - Área Total, Campo Nativo, Estrada, Mato Cultivado e Campo Limpo.

O layout da Tabela Geral para esse caso pode ser observado na Figura 10. Na parte inferior da referida figura é possível notar a presença de outras três planilhas:

- Quadro de Áreas onde se faz o comparativo entre a área de matrícula e a área obtida pelos cálculos;

- Campo A e Campo B onde se faz a ligaçáo com todas as tabelas.$d b f$ geradas pelo ArcGIS de acordo com a sua respectiva coluna, nesse caso: Campo Nativo, Estrada, Mata Nativa, Mato Cultivado e Campo Limpo.

Em seguida programa-se a Tabela Geral para que execute o erro de fechamento linear e distribua-o (caso exista) nas áreas de maior relevância para o cálculo final. Esta programação envolve que o software execute a diferença entre a Área Total da primeira coluna $(8,0001$ ha) (Figura 11)e o somatório das demais áreas. Esse cálculo verifica se os dados foram ou não corretamente calculados.

É válido ressaltar que o erro linear máximo admissível depende da qualidade da poligonal, a qual apresenta precisóes compreendidas entre 1/500 (baixa) até valores superiores a 1/100.000 (alta). Sendo assim, utilizando-se como exemplo uma poligonal com 1.000 m o erro máximo aceitável é $1 \mathrm{~m}$, ou seja, a precisão é de 1/1.000 (baixa) (PASTANA, 2016).

Em seguida para solucionar o erro, distribui-se a diferença encontradanas colunas com maior valor de área, pois a probabilidade de o erro ter ocorrido nessas áreas é, geralmente, maior do que nas demais. Para elucidar esse ponto, utiliza-se como o exemploo cálculo referente aConferência de Cálculoo qual é estimado em 100,0067\% ～(Figura 11). Como esse valor ultrapassa $100 \%$ a coluna Diferença apresenta um resultado negativo (-0,0067\%). Esse valor será incorporado nas colunas que tiverem a maior área (excluindo a primeira coluna referente à Área Total $8,0001 \mathrm{ha})$.

Para se montar as mencionadas colunas primeiramente calcula-se a área (em hectare) correspondente ao valor encontrado na coluna Diferença (\%). O valor resultante é expresso na coluna 


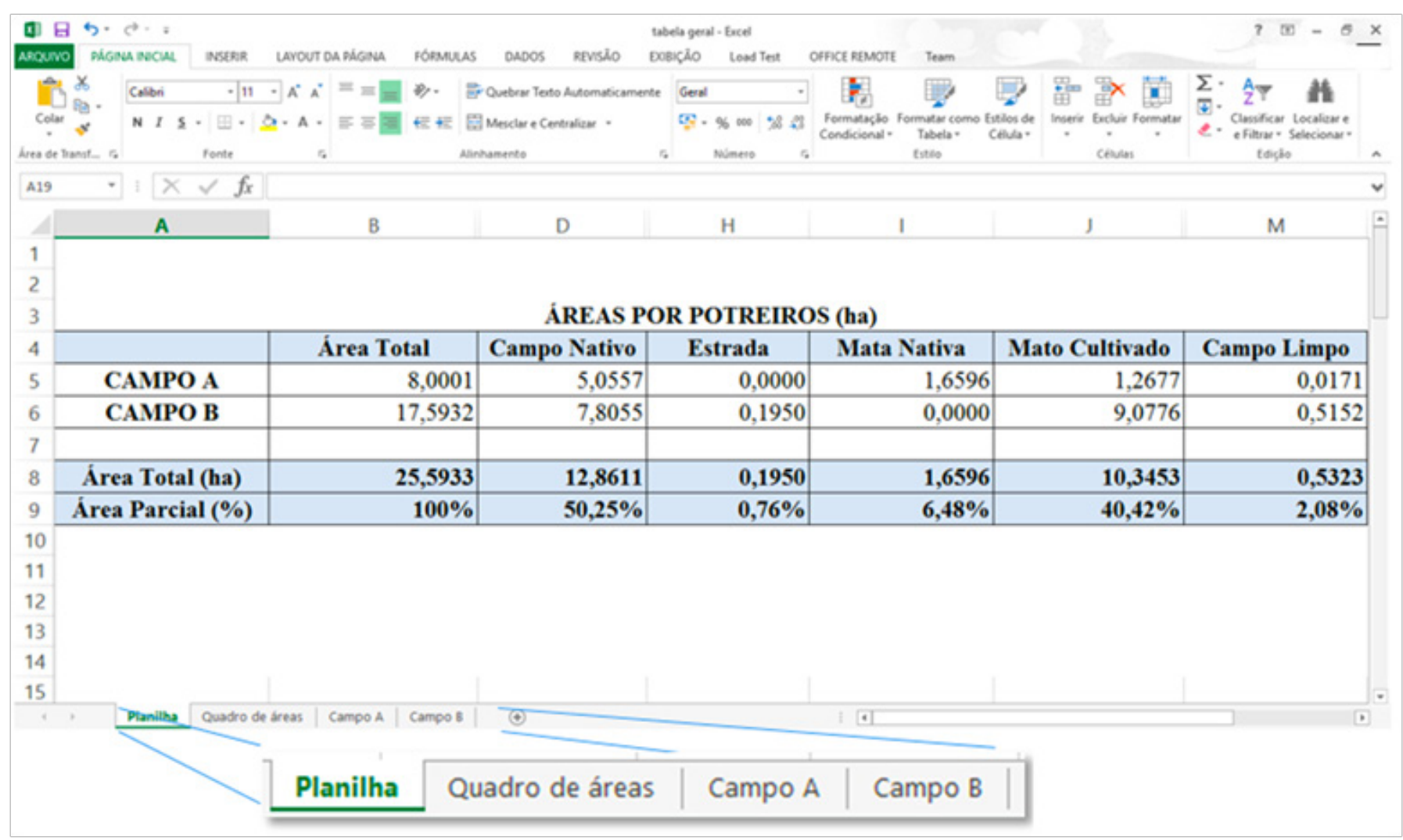

Figura 10.Layout da Tabela Geral: área com dois campos e cinco variáveis

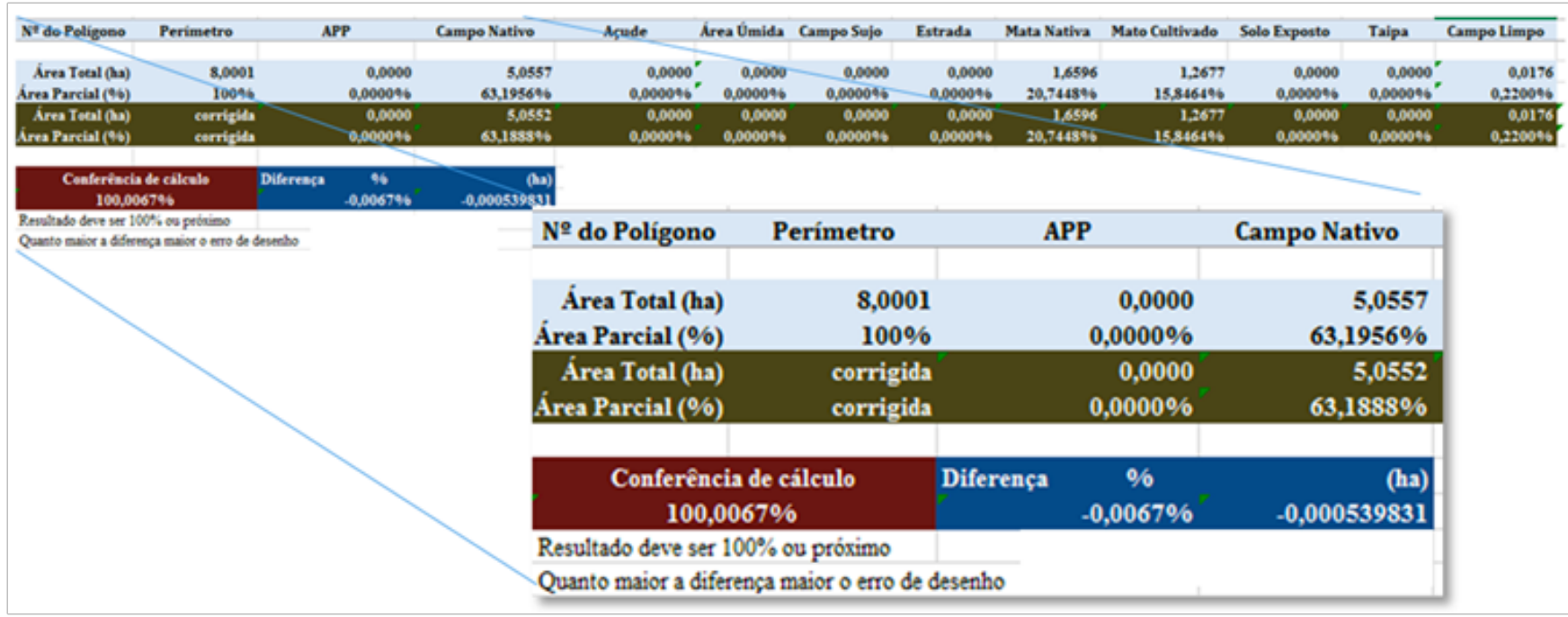

Figura 11.Layout da Tabela Geral: detalhamento da correção de erros

denominada ha. O qual é fruto do produto do valor percentual encontrado na coluna Diferença pela Área Total (8,0001 ha). O resultado de toda essa operação é somado ou subtraído nas colunas que têm a maior área. O novo resultado em hectareé visualizado na linha nomeada deÁrea Total (ha) corrigida. Já o resultado em porcentagem é feito adicionando ou subtraindo o resultado obtidona coluna Diferença com o resultado obtido na linha denominada de Área Parcial (\%) corrigida, optando-se nesse caso, por inseri-lo em uma única coluna a qual apresenta o maior valor de área.

Para a planilha referente ao Campo B o procedimento de cálculo é o mesmo.

Esse mesmo procedimento é seguido para automatizar o cálculo das áreas de mapas com números maiores de campos (A, B, C, D, E, F, G, H, ...). Todos eles possuem uma planilha individual que apresenta dados dos cálculos das áreas poligonais, os quais são agrupados em uma única planilha (Tabela Geral)a qual, ao final de todo o trabalho é inserida 


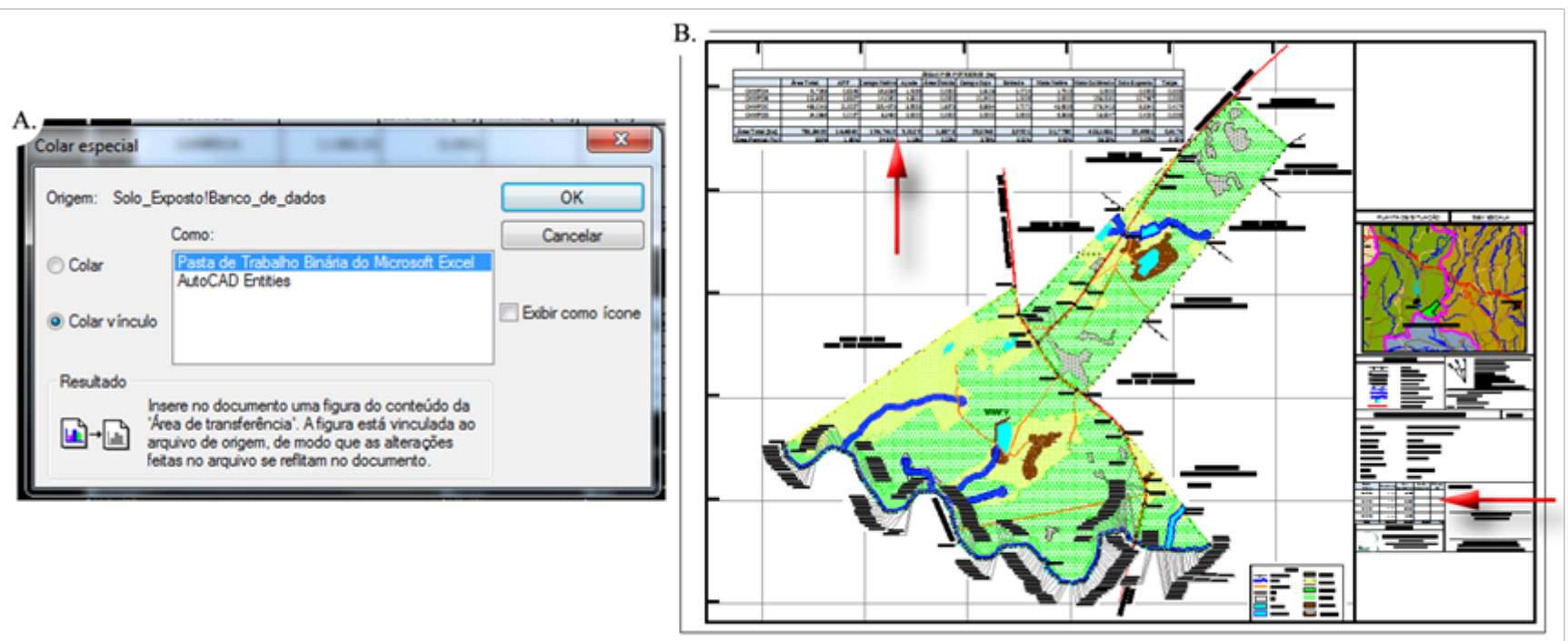

Figura 12. Inserindo a Tabela Geral e o Quadro de Áreas no AutoCAD: A. Colar especial; e B. Tabelas posicionadas na planta topográfica

no AutoCAD finalizando o ciclo de automatização e permitindo a interação entre os três tipos de software.

Para inserir a Tabela Geral no AutoCAD deve-se selecioná-la no Excel ir ao AutoCAD e escolherColar Especial (Figura 12A). Por fim posiciona-se a planilha no local desejado atribuindo-lhe os ajustes necessários (Figura 12B).

Para quantificar-se a eficiência do Método Automatizado frente ao Método Tradicional fez-se um comparativo utilizando-se cronometragem. O procedimento de avaliação parte de um mapa pronto com seus respectivos polígonos exportados. Repetiu-se o processo três vezes e fez-se uma média dos valores obtidos através da razão entre os tempos observados no Método Automatizado e os tempos observados no Método Tradicional

\section{RESULTADOS E DISCUSSÃO}

Vale ressaltar que no caso desse trabalho o levantamento foi feito com estação total e, portanto, não foi fonte de estudo ou comparação aos métodos de mediçôes e levantamentos remotos com restituiçôes aerofotogramétricas, restituiçôes por imagens orbitais, laser scanning terrestre e aerotransportado, os quais de acordo com o que diz Freitas (2011), se tornam cada vez mais presentespara complementoou até mesmo como alternativa no levantamento topográfico a campo

Com a análise descrita nesse artigo escreveu-se um manual de utilização o qual visa facilitar a implantação do procedimento descrito. Com esse manual em mãos, além de solucionar problemas operacionais é possível criar novas iteraçóes, aumentar o número de variáveis, mudar os modelos de mapas, replicar o processo de automatização, entre outras coisas (GARRÉ et al, 2016).

O sistema de interação com o usuário baseia-se na abertura de pastas e salvamento de arquivos. Sendo assim para fazer uso dessa programação o usuário deve acessar as pastas que compóe todos os modelos até o décimo campo (Figura 13A) escolhendo quantos campos o mapa necessitará ter (Figura 13B),ou seja, quantos campos precisarão ter suas áreas calculadas.

Cada modelo projetado é dotado de:

- Pastas individuais para cada campo;

- Planilha de cálculos gerais (Tabela Geral);

- Modelo em AutoCAD.

O Modelo em AutoCAD possui a planilha (Tabela Geral) inserida e vinculada ao Excel, bem como o Quadro de Áreas, conforme visualiza-se na Figura 14.

A metodologia de trabalho da empresa continua sendo a mesma descrita no subitem 2.2, porém agora o mapa base deve ser inserido no modelo em AutoCAD atentando-se para uma análise prévia de quantos campos o novo mapa terá. Caso sejam 4 campos, por exemplo, abre-se o mapa modelo presente na pasta nomeada de 4 Campos e insere-se o mapa base. Feito isso trabalha-se normalmente no novo mapa modelo 


\begin{tabular}{|c|c|c|c|c|c|}
\hline A. Nome & Data de modificaç... & Tipo & B. Nome & Data de modificaç... & Tipo \\
\hline 1] 1 Campo & 08/02/2016 14:48 & Pasta de arquivos & D. Campo A & $08 / 02 / 201614: 49$ & Pasta de arquivos \\
\hline 13. 2 Campos & 08/02/2016 14:48 & Pasta de arquivos & 1) Campo B & 08/02/2016 14:49 & Pasta de arquivos \\
\hline 13. 3 Campos & $08 / 02 / 201614: 48$ & Pasta de arquivos & 15. Campo C & 08/02/2016 14:49 & Pasta de arquivos \\
\hline (1) 4 Campos & $08 / 02 / 201614: 48$ & Pasta de arquivos & D) Campo D & 08/02/2016 14:49 & Pasta de arquivos \\
\hline 13. 5 Campos & 08/02/2016 14:48 & Pasta de arquivos & 질 Cálculos Gerais & 08/02/2016 14:53 & Planilha do Micro... \\
\hline 1] 6 Campos & 08/02/2016 14:48 & Pasta de arquivos & $\square$ Modelo Cad.bak & 08/02/2016 14:53 & Arquivo BAK \\
\hline D. 7 Campos & 08/02/2016 14:49 & Pasta de arquivos & As Modelo Cad & $08 / 02 / 201614: 53$ & AutoCAD Drawing \\
\hline 1) 8 Campos & 08/02/2016 14:49 & Pasta de arquivos & & & \\
\hline 1) 9 Campos & 08/02/2016 14:49 & Pasta de arquivos & & & \\
\hline 10 Campos & 08/02/2016 14:49 & Pasta de arquivos & & & \\
\hline
\end{tabular}

Figura 13. Sistema de interação com o usuário: A.Modelos pré-definidos; e B. Estrutura de cada modelo (modelo de 4 Campos)

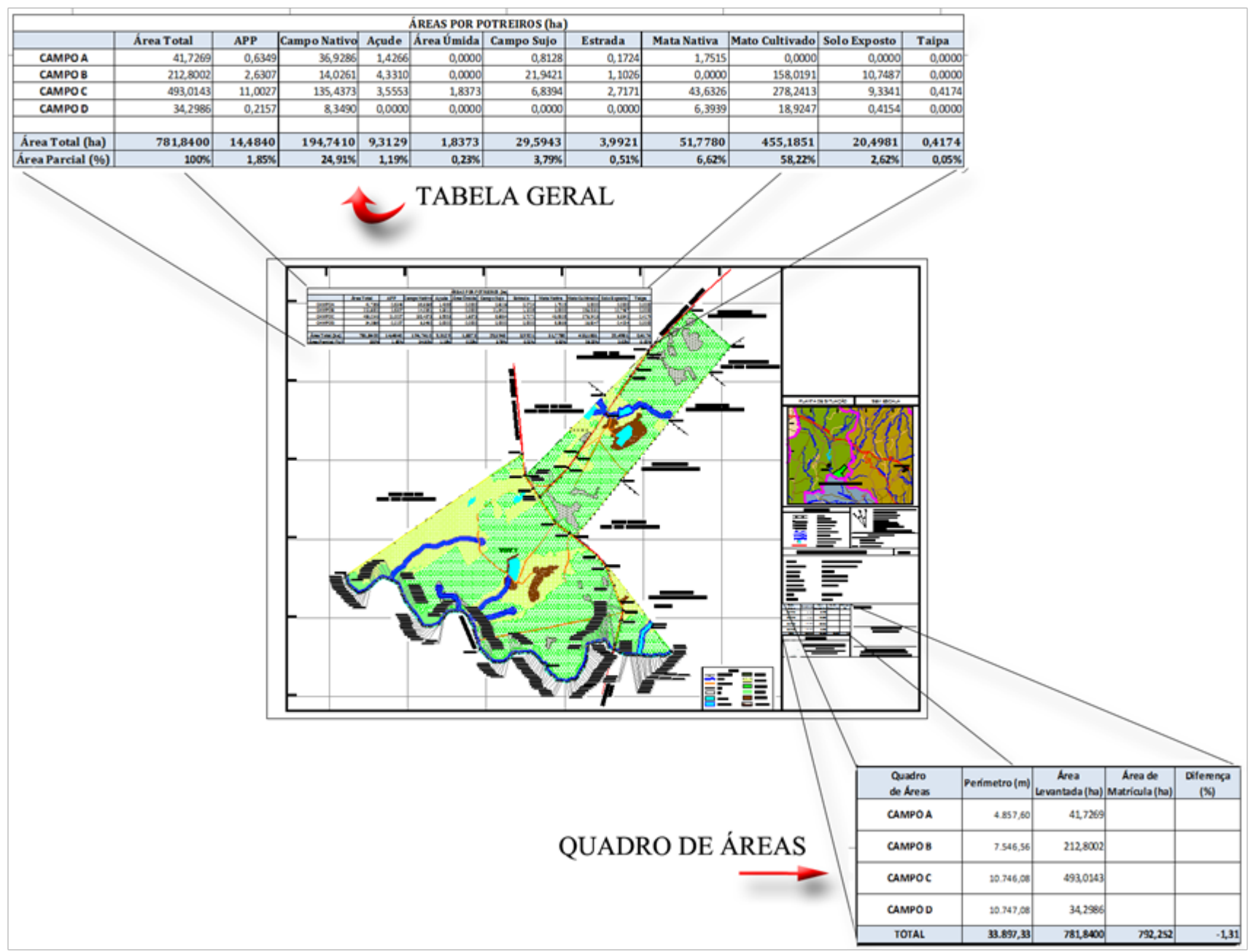

Figura 14. Layout final do Modeloem AutoCAD

gerando-se os polígonos referentes a cada tipo de área. Em seguida exportam-se esses polígonos, atentandose para que tenham os mesmos nomes contidos nos arquivos fontes (Campo_Nativo e APP, por exemplo) e ainda que sejam salvos no mesmo local de origem (Pastas com os nomes Campo A, B,C e D, nesse caso). Para que assim haja a substituição dos valores antigos pelos novos valores calculados (Excel)

Feito isso, todos os valores das planilhas intituladas de Tabela Geral e Quadro de Áreas se alteram, informando, assim, os novos valores e variáveis do novo mapa. Esse mapa agora pode ser salvo no mesmo local (pasta 4 Campos) ou ainda, ser salvo em um novo local com o nome da propriedade levantada (o mais 
indicado).

Uma deficiência nesse método é constatada por um erro de comunicação entre as planilhas do Excel que em algumas vezes faz com que os links da Tabela Geral inserida no AutoCAD não se atualizem. Para superar essa deficiência é necessário que o usuário, após calcular todos os valores de um campo abra todas as planilhas (.dbf) e em seguida abra o arquivo com nome Cálculos Gerais (Tabela Geral). Finalmente, salva-se o arquivo Cálculos Gerais e observa-se a modificação dos valores na Tabela Geral do AutoCAD.

A solução apresentada nesse artigo visa aumentar a eficiência na produção de mapas topográficos. Para isso, integrando-se o AutoCAD Map 3D ${ }^{\circledR}$, ArcGIS ${ }^{\circledast}$ e Excel ${ }^{\circ}$, automatizam-se os cálculos das áreas das poligonais representantes das características de uma dada propriedade

Por fim, a eficiência do processo foi de aproximadamente $67 \%$, sendo que para o Método Tradicional, desde a abertura do ArcGIS para importação dos polígonos e cálculo de área de cada um, o tempo médio cronometrado foi de 27 minutos e 36 segundos. Já para o Método Automatizado o tempo registrado foi de 9 minutos e 23 segundos. Esperam-se níveis de otimização maiores, caso o objeto de estudo seja um mapa de maior complexidade, pelo aumento no número de variáveis.

Como não se encontrou nenhuma referência que executasse a solução exposta neste artigo gera-se um manual de procedimentos o qual descreve, por intermédio de ilustraçóes explicadas, o passo-a-passo para que terceiros cheguem aos mesmos resultados evidenciados. Possibilitando que estes estudem e aprimorem o método aqui sintetizado.

\section{CONCLUSÃO}

O Método Automatizado mostra-se eficaz para reduzir o tempo destinado ao cálculo das áreas, possibilitando um aumento no número de mapas confeccionados no mesmo intervalo de tempo.

Os $67 \%$ de otimização verificados pelo Método Automatizado é o resultado médio averiguado para um mapa de complexidade média.

\section{REFERÊNCIAS}

ABNT NBR 13133 - Execuçáo de levantamento topográfico. Disponível em: <http://www.carto.eng.uerj.br/cdecart/ download/NBR13133.pdf>. Acesso em: 11 set. 2016.

ARCGIS/ARCMAP - Fundamentos de cálculos de campo. Disponível em: <http://des ktop.arcgis.com/en/arcmap/10.3/ manage-data/tables/fundamentals-of-field-calculations.htm>. Acesso em: 11 set. 2016.

BUENO, R.;Consciência profissional. A importância de identificar as características que influenciam nos serviços de agrimensura e cartografia. Editora MundoGEO. Revista InfoGNSS. Ano 6, Edição n 27, 2009. 40 p.

CADBLOG. Importar tabela do Excel para o AutoCAD. Disponível em:<http://cadblog2010.blogspot.com.br/2011/05/ importar-tabela-do-excel-para-oautocad.html>. Acesso em: 26 jun. 2016.

D’ALGE, J. C. L;Geoprocessamento - Teoria e Aplicaçóes Parte I - Cap. 6 - Cartografia para Geoprocessamento. INPE Instituto Nacional de Pesquisas Espaciais, 2001. Disponível em <www.dpi.inpe.br/gilberto/livro/introd/>. Acesso em 10 ago. 2016.

FILHO, A.C.; CARNEIRO, A.F.T.;AYALA, C.; CICCARONE, C.; BULHÓES, D. B.; SANTOS, F. L. A.; MARINATO, F.; OLIVEIRA, G. G.; GERMANI, G. I.; RAMOS, I. C. A.; TAGLIAPIETRA, J. R. C.;CAVIGNAC, J.; MITIDIERI, L.; JOB, J.; RIOS, M.; MARTINS, O. O.; BORTOLETTO, R. S.; SILVA, S. J.; LIMA, S. H. S.; FERREIRA, S. R. B.; NICHOLS, S.; FEHLAUER, T.INCRA e os Desafios para a regularização dos territórios Quilombolas: algumas experiências.MDA/ INCRA, Brasília - DF, 2006. 186 p.

FREITAS, E.; Imagens Invadem o Campo. Dados de satélite, nuvem de pontos $3 \mathrm{D}$ e fotos aéreas cada vez mais presentes nos levantamentos. Editora MundoGEO. Revista InfoGNSS. Ano 6, Edição n 35, 2011. 26 a 28 p.

GARRÉ, S. O.; WEYMAR, G. J.; CHAGAS, M. A.; Procedimentos para Automatizar o Cálculo de Áreas Topográficas.Editora Clube dos Autores. Edição no 1, 2016, 56p. GEOBRAINSTORMS. Interaçáo ArcGIS/AutoCAD Parte II. Disponível em:<http://geobrainstorms.wordpress. com/2011/09/28/interacao-arcgisautocad-parte-2/>. Acesso em: 28 jun. 2016.

IBGE - Instituto Brasileiro de Geografia e Estatística. Introdução à Geodésia. Disponível em: <http://www.ibge.gov.br/home/ geociencias/geodesia/default.shtm> Acesso em: 06 set. 2016.

INCRA - Instituto Nacional de Colonização e Reforma Agrária.

Certificado de Cadastro de Imóvel Rural - CCIR/INCRA.

Disponível em:<http://www.incra.gov.br/sncr_ccir $>$. Acesso em: 26 jun. 2016.

INPE - Instituto Nacional de Pesquisas Espaciais. Introdução à Ciência da Geoinformaçáo, 2001. PDF Online, 345 p. Disponível em: <http://mtcm12.sid.inpe.br/col/sid.inpe.br/ sergio/2004/04.22.07.43/doc/publicacao.pdf> Acesso em: 02fev. 2016.

MDA - Ministério do Desenvolvimento Agrário.Norma Técnica para georeferenciamento de imóveis rurais. 3a Ediçáo. Disponível em:<http://www.incra.gov.br/media/institucional/ norma $\% 20$ tecnica $\% 20$ para $\% 20$ georreferenciamento $\% 20$ de $\% 20$ imoveis $\% 20$ rurais $\% 203 \% 20$ edi $\%$ C3\%A7ao.pdf $>$. Acesso em: 28 jun. 2016. 
MOURA, A. C. M.;Geoprocessamento na Gestão e Planejamento Urbano. Belo Horizonte, Ed. Da Autora, 2003. 294 p.

PASTANA, C. E. T. Topografia I e II. Anotaçóes de Aula. Paraná. UNIMAR-FEAT, 2010. Apostila, 255 p.
VALERIANO, M. M.;Modelo digital de elevaçáo com dados SRTM disponíveis para a América do Sul. São José dos Campos, SP: INPE: Coordenação de Ensino, Documentaçáo e Programas Especiais (INPE-10550-RPQ/756), 2004. 72p. 